\title{
Assessment of Slope-Adaptive Metrics of GEDI Waveforms for Estimations of Forest Aboveground Biomass over Mountainous Areas
}

\author{
Wenjian Ni ${ }^{1},{ }^{1,2}$ Zhiyu Zhang, ${ }^{1}$ and Guoqing Sun $^{3}$ \\ ${ }^{1}$ State Key Laboratory of Remote Sensing Science, Aerospace Information Research Institute, Chinese Academy of Sciences, \\ Beijing 100101, China \\ ${ }^{2}$ University of Chinese Academy of Sciences, Beijing 100049, China \\ ${ }^{3}$ Department of Geographical Sciences, University of Maryland, College Park, MD 20742, USA
}

Correspondence should be addressed to Wenjian Ni; niwj@radi.ac.cn

Received 18 February 2021; Accepted 1 August 2021; Published 27 August 2021

Copyright (C) 2021 Wenjian Ni et al. Exclusive Licensee Aerospace Information Research Institute, Chinese Academy of Sciences. Distributed under a Creative Commons Attribution License (CC BY 4.0).

\begin{abstract}
Waveform broadening effects of large-footprint lidar caused by terrain slopes are still a great challenge limiting the estimation accuracy of forest aboveground biomass (AGB) over mountainous areas. Slope-adaptive metrics of waveforms were proposed in our previous studies. However, its validation was limited by the unavailability of enough reference data. This study made full validation of slope-adaptive metrics using data acquired by the Global Ecosystem Dynamics Investigation (GEDI) mission, meanwhile exploring GEDI waveforms on estimations of forest AGB. Three types of waveform metrics were employed, including slope-adaptive metrics (RHT), typical height metrics relative to ground peaks (RH), and waveform parameters (WP). In addition to terrain slopes, two other factors were also explored including the geolocation issue and signal start and ending points of waveforms. Results showed that footprint geolocations in the first version GEDI data products were shifted to the left forward of nominal geolocations with a distance of about $24 \mathrm{~m} \sim 30 \mathrm{~m}$ and were substantially corrected in the second version; the fourth and fifth groups of signal start and ending points of waveforms had worse performance than the rest of the four groups because they used the maximum and minimum signal thresholds, respectively. Taking airborne laser scanner (ALS) data as reference, the root mean square error (RMSE) of terrain slopes extracted from the digital elevation model of the shuttle radar topography mission (SRTM DEM) was about $3^{\circ}$. The coefficients of determination $\left(R^{2}\right)$ of estimation models of forest AGB based on RH metrics were improved from 0.48 to 0.68 with RMSE decreased from $19.7 \mathrm{Mg} / \mathrm{ha}$ to $15.4 \mathrm{Mg} / \mathrm{ha}$ by the second version geolocations. The RHT and WP metrics gave the best and the worst estimation accuracy, respectively. RHT further improved $R^{2}$ to 0.77 and decreased RMSE to $13.0 \mathrm{Mg} /$ ha using terrain slopes extracted from SRTM DEM with a resolution of $1 \mathrm{arc}$ second. $R^{2}$ of estimation models based on RHT was finally improved to 0.8 with RMSE decreased to $11.7 \mathrm{Mg} / \mathrm{ha}$ using exact terrain slopes from ALS data. This study demonstrated the great potential of slope-adaptive metrics of GEDI waveforms on estimations of forest aboveground biomass over mountainous areas.
\end{abstract}

\section{Introduction}

Accurate accounting of forest carbon storage in vegetation is essential for global carbon budgets. According to the results of global carbon budgets, the imbalance item for the last decade is $0.4 \mathrm{GtCyr}^{-1}$, which is about $26.6 \%$ of carbon emissions from land-use change [1]. The budget imbalance is caused by the overestimation of carbon sources and/or the underestimation of carbon sinks. The role of remote sensing in the accounting of global carbon budgets has been widely recognized, and several regional remotely sensed estimations/maps have been released [2-5]. However, huge inconsistency among them was reported using multiple reference datasets [6]. These estimations still are not based on the measurement of variables determining the forest carbon storage but only a kind of combination of landcover types and representative carbon values [7]. Although measurements provided by spaceborne lidar are employed, these wall-to-wall maps were mostly produced relying on multispectral reflectance and/or radar backscattering coefficients 
due to the lack of imaging capability of the spaceborne lidar. The limited capability of either the multispectral reflectance or the radar backscattering coefficients on the quantifying of carbon storage has been widely demonstrated due to the lack of physical basis [8] and is always restricted by the sensitivity saturations and terrain effects $[9,10]$.

The forest carbon storage in vegetation is directly determined by forest spatial structures depicted by tree sizes, stem densities, and forest types. The measurement of forest spatial structures is the thorough solution for the accurate accounting of carbon storage. The multispectral reflectance and/or radar backscattering coefficients are not the direct measurement of forest spatial structures and are easily affected by environmental factors. Therefore, the new generation remote sensing satellites focusing on carbon storage and its dynamics rely on the technology of either lidar or the polarimetric SAR interferometry (PolInSAR), such as the mission of Global Ecosystem Dynamics Investigation (GEDI) [11] and BIOMASS [9]. The synthesis of lidar with PolInSAR is promising for the accurate mapping of carbon storage at global scales through the direct detection of forest spatial structures [12].

Spaceborne large-footprint lidar plays an important role in the existing global maps as well as the near-future remote sensing schemes of forest carbon storages. As the successor of GEDI, the mission of Japanese vegetation lidar (MOLI) will continue on the mapping of forest aboveground biomass (AGB) on a global scale [13]. China is scheduled to launch their first scientific experimental satellite dedicated to the comprehensive monitoring of terrestrial ecosystems in 2022, i.e., the Terrestrial Ecosystem Carbon Monitoring Satellite (TECIS-1). One of the main instruments onboard TECIS-1 is a three-beam large-footprint waveform lidar [14]. In fact, China has launched their large-footprint lidar systems in 2019 [15], i.e., the seventh satellite in their high-resolution earth observation system (GF-7), although it is not specifically designed for forest monitoring and its pulse rate is only $3 \mathrm{~Hz}$.

The performance of large-footprint lidar on the sampling of forest spatial structures has been well recognized on flat terrains over the past two decades, due to its capability of the direct detection of forest spatial structures using laser ranging. However, the biggest challenge limiting the estimation accuracy of forest AGB over mountainous areas is the waveform broadening effect caused by terrain slopes, i.e., the canopy and ground returns tend to be stretched and mixed up over steep topographies. It is reported that $23 \%$ of global forest cover is distributed over mountainous areas [16], and $57.6 \%$ of forest cover with terrain slope greater than $15^{\circ}$ is in China [17]. Much effort has been devoted to solving this issue including the fully empirical corrections [18] as well as physically based methods [19]. Given that the global elevation datasets are increasing [20-23], we proposed slope-adaptive metrics of lidar waveforms by referencing the calculated bare ground waveform with known terrain slopes [24]. The validation of slopeadaptive metrics using simulated waveforms indicated the great potential to make better biomass estimations than those using metrics based on the Gaussian decompositions and lead and trail metrics extracted from waveforms. However, only limited numbers of real waveforms were used in the validation due to the unavailability of reference data over footprints of real waveforms.

The global dataset acquired by GEDI after April 18, 2019, has been released since February 07, 2020. It provides a great opportunity to make a full assessment of slopeadaptive metrics. In order to make full validation of slopeadaptive metrics using data acquired by the GEDI mission, meanwhile exploring GEDI waveforms on estimations of forest AGB, this study reports the assessment of slopeadaptive metrics of waveforms using GEDI data for estimations of forest aboveground biomass over mountainous areas.

\section{Materials}

2.1. Test Sites. The test site chosen in this study is the Cibola National Forest on Sandia Mountains $\left(35^{\circ} 12^{\prime} 41.39^{\prime \prime} \mathrm{N}\right.$, $106^{\circ} 24^{\prime} 56.91^{\prime \prime}$ W) immediately to the east of the city of Albuquerque in New Mexico in the southwestern United States as shown in Figure 1(a). The elevation ranges from $2252 \mathrm{~m}$ to $3149 \mathrm{~m}$ as shown in Figure 1(b). The distributions of terrain slopes are $0-5^{\circ}: 4.0 \%, 5-15^{\circ}: 43.1 \%, 15-30^{\circ}: 41.4 \%$, and $>30^{\circ}: 11.5 \%$. The dominant tree species include Douglas fir (Pseudotsuga menziesii), Ponderosa pine (Pinus ponderosa), Pinyon pine (Pinus edulis), Rocky mountain juniper (Juniperus scopulorum), Bristlecone pine (Pinus aristata), Blue spruce (Picea pungens), and Engelmann spruce (Picea engelmannii). In addition, there are several other species such as alder, willow, and maple. Tree heights range from 0.0 to $35.0 \mathrm{~m}$ as shown in Figure 1(c).

2.2. G-LiHT Data. The reference forest AGB collected over mountainous areas is necessary for the assessment of slope-adaptive metrics. However, it is difficult to collect enough reference data by field measurements, especially over steep terrain conditions. The forest AGB estimated using point cloud acquired by an airborne laser scanner (ALS) is used as reference data in this study. The ALS data was collected on June $25^{\text {th }}, 2018$, by Goddard's LiDAR, Hyperspectral \& Thermal Imager (G-LiHT) [25] for the forest health protection founded by the US Forest Service (USFS). The area is about $9.0 \mathrm{~km}$ in length and $2.9 \mathrm{~km}$ in width. The nominal flight height is about $335 \mathrm{~m}$ above ground level with a velocity of $200-270 \mathrm{~km} / \mathrm{h}$. The laser pulse repetition frequency is $300 \mathrm{kHz}$, and the scan rate is 150 lines/second. There are 8 returns per pulse maximally recorded. The point density is about 30 points $/ \mathrm{m}^{2}$. The positional accuracy is smaller than $10 \mathrm{~cm}$ horizontal and $20 \mathrm{~cm}$ vertical. The points within the GEDI footprint are extracted from the G-LiHT point cloud. These points have been classified as ground and nonground by the data provider. The ground surface elevation model is built on ground points. Nonground points are normalized by their corresponding ground surface elevations. The reference forest AGB was calculated using the normalized points and the prediction model developed by Nelson et al. [26], of which $R^{2}$ is 0.71 , which is acceptable to serve as reference data to make comparisons 


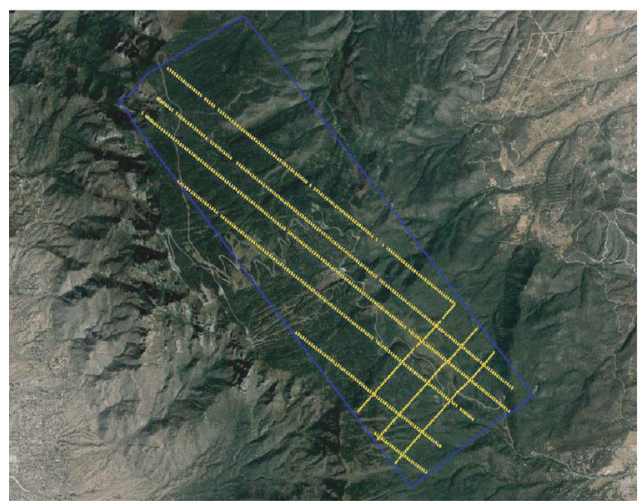

(a)

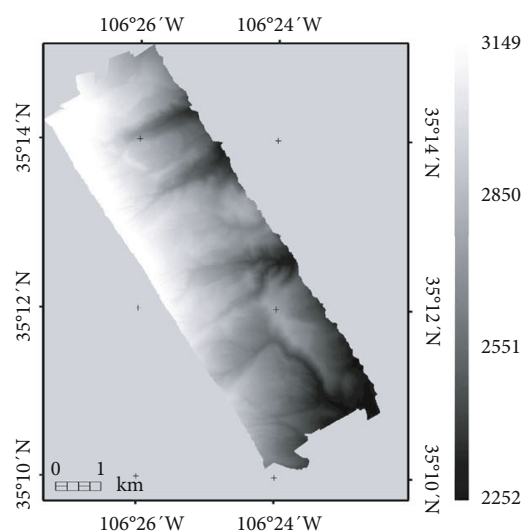

(b)

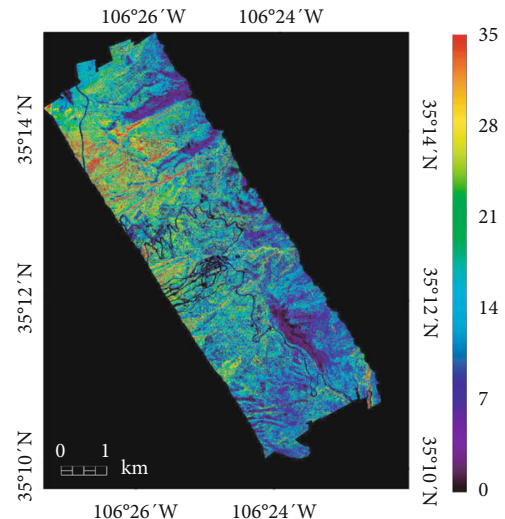

(c)

FIGURE 1: Spatial coverage and terrain conditions of test sites: (a) spatial coverage of G-LiHT (blue polygon) and GEDI data (yellow dotted lines) on true-color image from Google Earth; (b) terrain elevations from G-LiHT; (c) forest canopy height model from G-LiHT.

of the performance of different waveform metrics, although it may not be as accurate as those specifically developed based on the field measurements of forest plots if we have them.

2.3. GEDI Data. Two GEDI datasets came across the study area as shown by yellow lines in Figure 1(a). One was acquired around 6:16:00 am on August $21^{\text {st }}, 2019$, on the ascending orbit, i.e., southwest to northeast, while the other was acquired around 6:40:00 am on September $7^{\text {th }}, 2019$, on the descending orbit (i.e., northwest to southeast). They are referred to as the first and second dates in this study. The nominal footprint size is about $25 \mathrm{~m}$ in diameter. The three beams of the first date data are "0011," "0101," and "0110" from left to right (or from north to south), and 142 waveforms were collected within the ALS spatial coverage. The six beams of the second date data are "0000," "0001," “0010," “0011," “0101," and "0110" from left to right (or from north to south), and 602 waveforms were collected. Two versions of GEDI datasets have been released. It is documented that the second version has better geolocation accuracy than the first one. Both versions of GEDI datasets are used in this study to observe their geolocation differences. For convenience, the first and second version GEDI data are referred to as nominal and updated data in the following text, respectively.

\section{Methods}

3.1. Optimization of GEDI Footprint Geolocations. For the assessment of slope-adaptive metrics, the terrain slope should be extracted from the existing global elevation datasets and the reference data should be extracted from the G-LiHT data. Their key link is geolocation. It is reported that the positioning accuracy of the nominal data is within $15 \sim 20 \mathrm{~m}$ in terms of standard deviation [11, 27]. Therefore, the geolocation accuracy of GEDI footprints is an important issue for this study.

It has been widely accepted that the match between simulated waveforms based on point cloud and real waveforms is an effective way to refine the waveforms' geolocation $[28,29]$. In this study, the latitude and longitude of the lowest mode given in the L2A product are taken as the nominal geolocation of a waveform. The simulated locations shift easting and northing $\pm 50 \mathrm{~m}$ about the nominal geolocation with increments of $2.0 \mathrm{~m}$. The point cloud is extracted according to the shifted position and field of view of the laser beam. The simulated waveform is the summation of each point convoluted with a transmitted laser pulse given in the L1B products and weighted by the Gaussian function according to its horizontal distance to the beam center. The simulated waveform is finally normalized according to the background noise given in the L2A products and the biggest waveform amplitude. The match of shapes between GEDI and simulated waveforms is used to adjust the geolocation of each footprint. The resultant geolocations are referred to as optimized geolocations.

3.2. Extraction of Slope-Adaptive Metrics Using GEDI. Slopeadaptive metrics are defined as the difference between the percentile metrics of the GEDI waveform and the corresponding ones of the bare ground waveform with a given terrain slope on the footprint [24]. Supposing the Gaussian waveform of a bare ground is cut off by 2 times of the standard deviation, the slope-adaptive metrics can be mathematically expressed as follows:

$$
\begin{gathered}
\mathrm{RHT}_{i}=H_{i}-H g_{i}, \\
H_{i}=E_{i}-E_{e},
\end{gathered}
$$

where $\mathrm{RHT}_{i}$ is the slope-adaptive metrics, $H_{i}$ is the percentile metrics of the GEDI waveform referencing to its signal ending point, $\mathrm{Hg}_{i}$ is the percentage metrics of bare ground waveform referencing to its signal ending point, $E_{i}$ is the elevation where the accumulated waveform energy reaches a percentage of the total energy, and $E_{e}$ is the elevation of signal ending points. If $f(x)$ is the GEDI waveform and $E_{s}$ is 
the elevation of the signal starting point, the elevation of $E_{i}$ can be determined by

$$
\sum_{E_{e}}^{E_{i}} f(x) \Delta_{x}=i \sum_{E_{e}}^{E_{s}} f(x) \Delta_{x}, \quad E_{e} \leq E_{i} \leq E_{s}, 0 \leq i \leq 1
$$

$H_{i}$ is provided in the GEDI L2A products named as rx_cumulative. For $H g_{i}$, if $g(x)$ is the waveform model of the bare ground with a standard deviation of $\sigma_{1}$, a total extent from $-2 \sigma_{1}$ to $2 \sigma_{1}$ and the $-2 \sigma_{1}$ is aligned with $E_{e}$ of the waveform; it can be calculated as follows:

$$
\begin{aligned}
H g_{i} & =p_{i}+2 \sigma_{1}, \\
\sum_{-2 \sigma_{1}}^{p_{i}} g(x) \Delta_{x} & =i \sum_{-2 \sigma_{1}}^{+2 \sigma_{1}} g(x) \Delta_{x}, \quad-2 \sigma_{1} \leq p_{i} \leq 2 \sigma_{1}, 0 \leq i \leq 1, \\
g(x) & =\exp \left(\frac{-x^{2}}{2 \sigma_{1}^{2}}\right), \quad \sigma_{1}=\sigma_{0}+0.5 * \gamma * \tan (\theta),
\end{aligned}
$$

where $\gamma$ is the radius of the footprint, $\theta$ is the given terrain slope, $\sigma_{0}$ is the standard deviation of the transmitted Gaussian laser pulse, and $p_{i}$ is the ending point of integration in the calculation of $\mathrm{Hg}_{i}$.

Several global elevation datasets have been published including SRTM DEM [20], ASTER GDEM [30], TandemX DEM [31], and AW3D30 [32]. These datasets are different kinds of digital surface models derived from different types of remote sensing imagery, but none of them is the true elevation of the ground surface. Therefore, they are mostly affected by land cover types, more or less. Considering that most of them are the compiled results based on observations of several years, SRTM has the narrowest time span, i.e., Feb. $11^{\text {th }}-22^{\text {nd }}, 2000$. Therefore, the terrain slope is extracted from the SRTM DEM with a resolution of 1.0 arc second.

3.3. Other Waveform Metrics. The other two types of waveform metrics are also evaluated for the estimation of forest AGB in this study, in addition to the slope-adaptive metrics. One type is the widely used metrics defined by referencing to the ground peak. Although it is difficult to identify the true ground peak, the L2A products of GEDI provided the position of the lowest peak (mode). The metrics can be mathematically expressed as follows:

$$
\mathrm{RH}_{i}=E_{i}-E_{l},
$$

where $E_{l}$ is the elevation of the lowest mode provided in the GEDI L2A products.

The other type is the waveform parameters including the waveform extent, leading edge extent, and trailing edge extent defined in Lefsky [18], which can be mathematically expressed as follows:

$$
\text { extent }=E_{s}-E_{e} \text {, }
$$

$$
\begin{aligned}
& \text { trail10 }=E_{i}-E_{e}, \quad i=0.1, \\
& \text { lead10 }=E_{s}-E_{i}, \quad i=0.9 .
\end{aligned}
$$

3.4. Analysis Scheme. Three issues have to be addressed in the assessment of slope-adaptive metrics of GEDI on the estimation of forest AGB. The first one is the geolocations used in the extraction of the reference data from G-LiHT, which is evaluated by four cases: (a) case I: the nominal geolocations of first version GEDI L2A products; (b) case II: the updated geolocations from the second version GEDI L2A product; (c) case III: nominal geolocations of GEDI footprints adjusted by the mean shift of all footprints of each date data, i.e., optimized geolocations; and (d) case IV: the detailed shift of each footprint.

According to the definitions, the calculation of slopeadaptive metrics is based on the signal start and ending points. The L2A products provide six groups of signal start and ending points based on different smooth widths and thresholds. Therefore, the second one to be evaluated is the effects of the signal start and ending points. This study separately evaluates slope-adaptive metrics extracted based on these six groups of signal start and ending points.

The third one to be evaluated is the effects of terrain conditions. Most studies have demonstrated that the terrain slope of $15^{\circ}$ is a critical point. Therefore, GEDI waveforms are divided into two groups with terrain slopes below and above $15^{\circ}$, respectively. In addition, terrain slopes extracted from SRTM DEM and G-LiHT data are evaluated separately.

\section{Results}

4.1. Effects of Geolocation Optimization. Figure 2 shows simulated waveforms using the G-LiHT point cloud extracted by nominal, updated, and optimized geolocations of footprints against real GEDI waveforms as examples. The $x$-axis and $y$-axis are the amplitude and elevation of GEDI waveforms, respectively. The red line and blue dots are simulated and real waveforms, respectively. The simulated waveform is normalized by the noise and the biggest peak of GEDI waveforms. The first and second rows are the simulated waveforms of nominal and updated geolocations given in the lowest mode in L2A products, respectively, while the third row is that of optimized geolocations. It can easily be seen that there are apparent differences between simulated waveforms by nominal geolocations and GEDI waveforms in terms of both elevation ranges and shapes as shown in Figures 2(a)-2(c). Figures 2(d)-2(f) show an obvious improvement in the updated data. The optimized geolocations give the best match as shown in Figures 2(g)-2(i), although the waveform details do not match very well.

Figure 3 shows spatial distributions of updated and optimized geolocations of GEDI footprints relative to nominal ones. For the first date, i.e., those acquired on August $21^{\text {st }}$, 2019 , on the ascending orbit, the shifts along the $x$-axis are negative while those along the $y$-axis are positive as shown in Figure 3(a). That means real positions of GEDI footprints should be on the northwest side of nominal geolocations. 


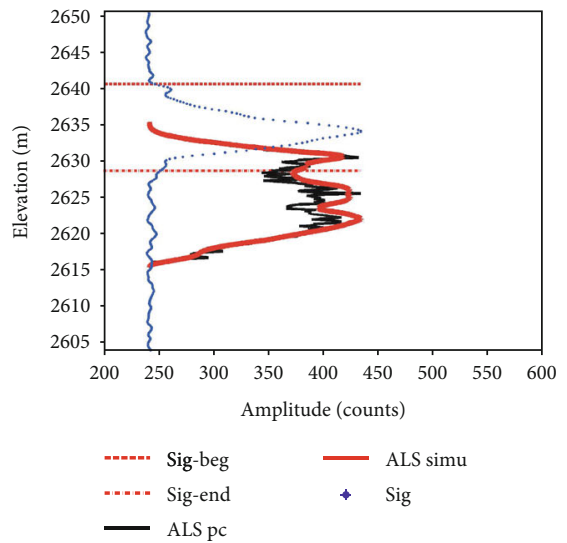

(a)

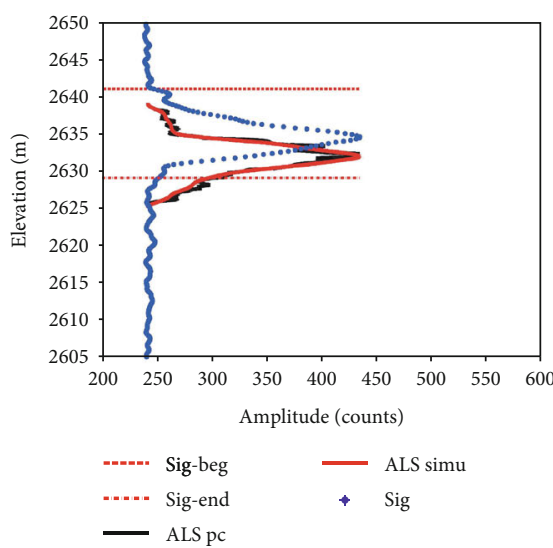

(d)
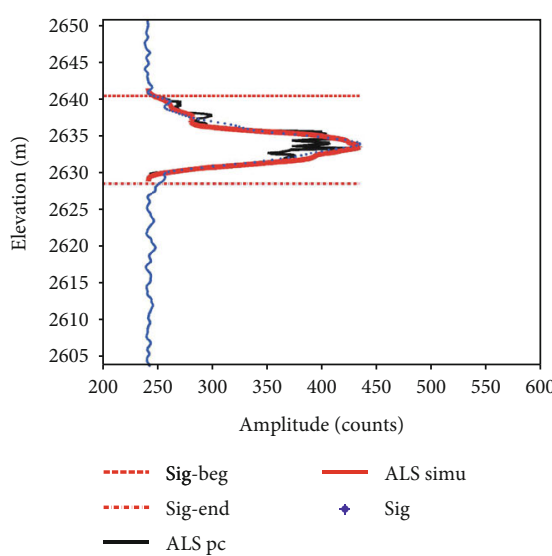

(g)

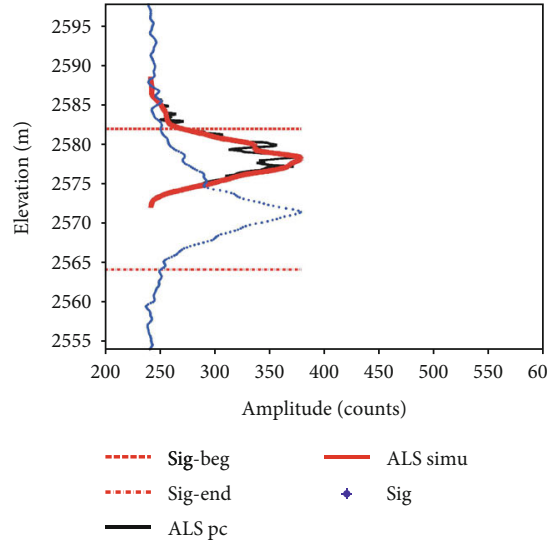

(b)

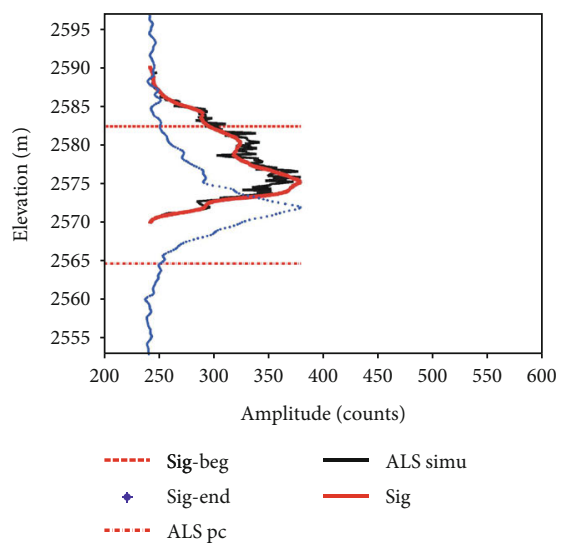

(e)
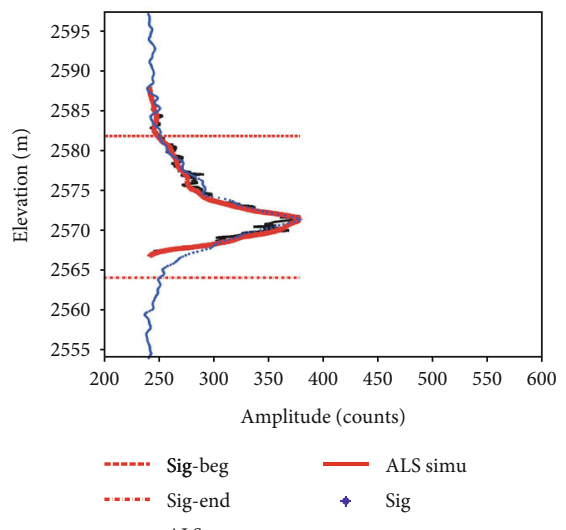

(h)

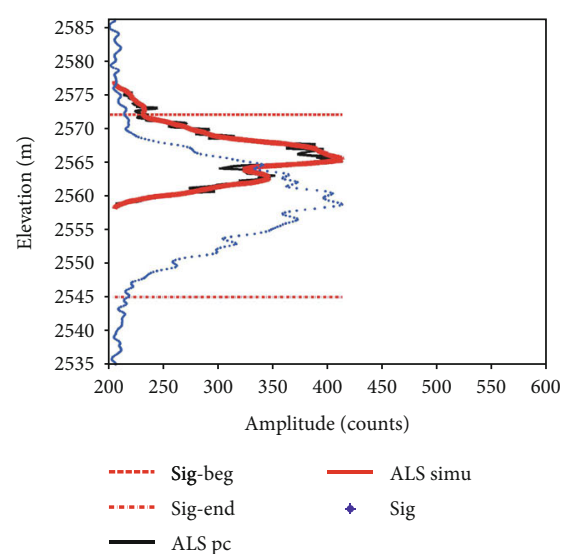

(c)

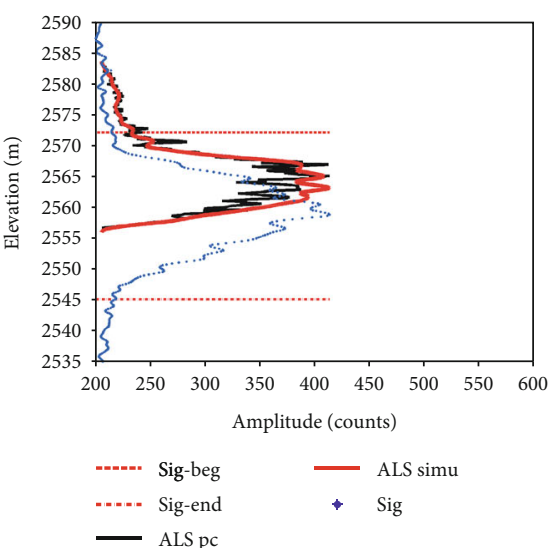

(f)

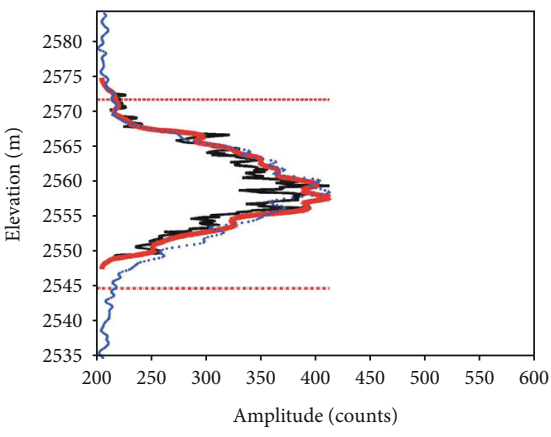

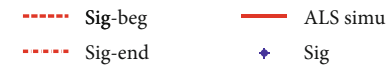

(i)

FIGURE 2: GEDI real and simulated waveforms using G-LiHT point cloud extracted by footprints with nominal, updated, and optimized geolocations: (a-c) nominal geolocations; $(d-f)$ updated geolocations; (g-i) optimized geolocations.

The average shifts are $-12.8 \mathrm{~m}$ and $20.4 \mathrm{~m}$ along the $x$-axis and $y$-axis, respectively. The standard deviation of distances to the average center is about $3.8 \mathrm{~m}$. For the second date, i.e., those acquired on September $7^{\text {th }}, 2019$, on the descending orbit, both shifts along the $x$-axis and $y$-axis are positive, which means the shift is to the direction of northeast. The average shifts are $24.3 \mathrm{~m}$ and $18.5 \mathrm{~m}$ along the $x$-axis and $y$-axis, respectively. The standard deviation of distances to the mean center is about $4.7 \mathrm{~m}$. Figures $3(\mathrm{c})$ and $3(\mathrm{~d})$ show the shift of updated geolocations to the nominal ones. The average shifts of the first date data are $-9.9 \mathrm{~m}$ and $7.8 \mathrm{~m}$ along the $x$-axis and $y$-axis, respectively, while they are $15.2 \mathrm{~m}$ and $16.1 \mathrm{~m}$ for the second date data. It can be seen that the updated ones shift to the same direction as the optimized ones. That means the position accuracy is indeed improved in the updated version compared with the nominal ones. Taking the average center of optimized geolocations as a reference, the position errors are reduced from $24.1 \mathrm{~m}$ to 


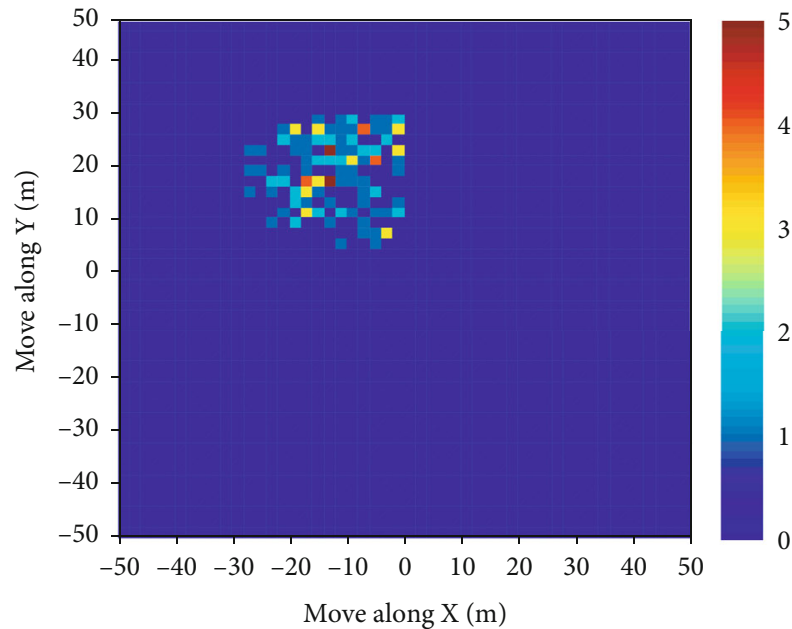

(a)

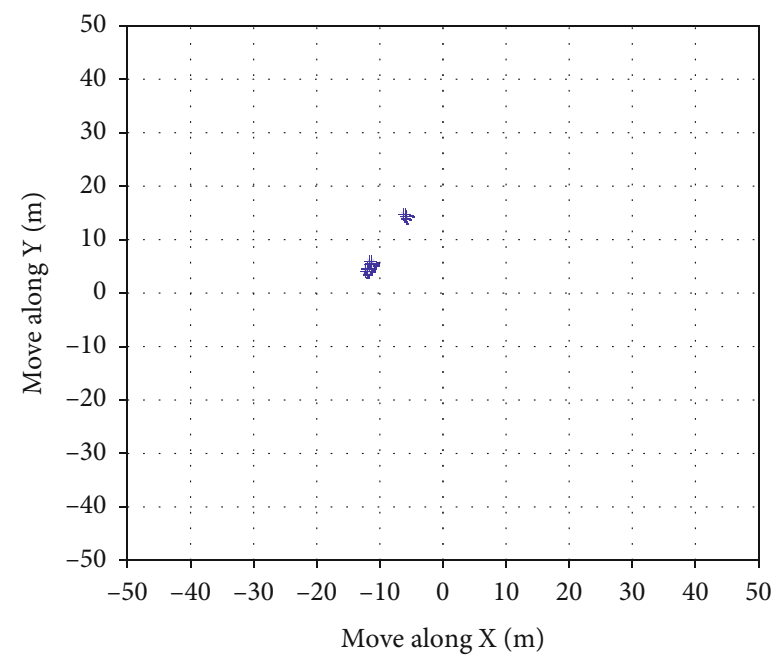

(c)

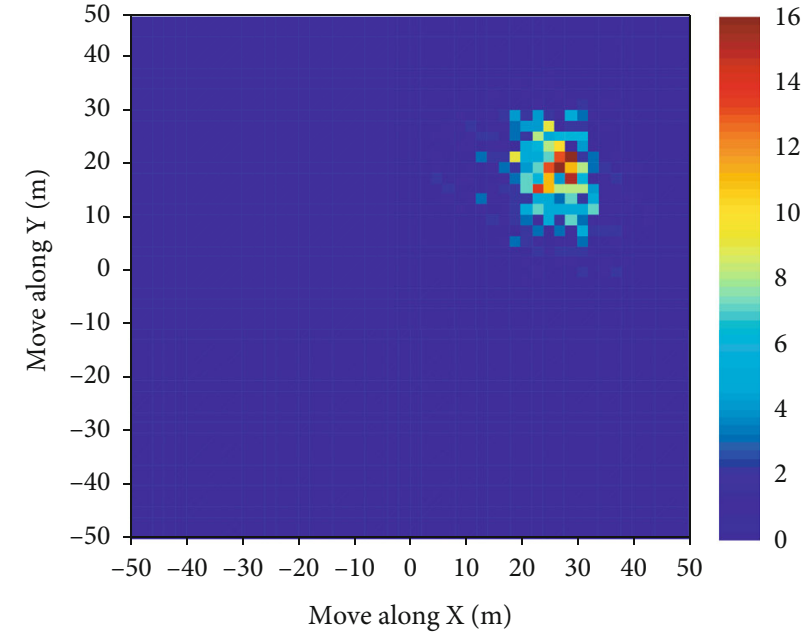

(b)

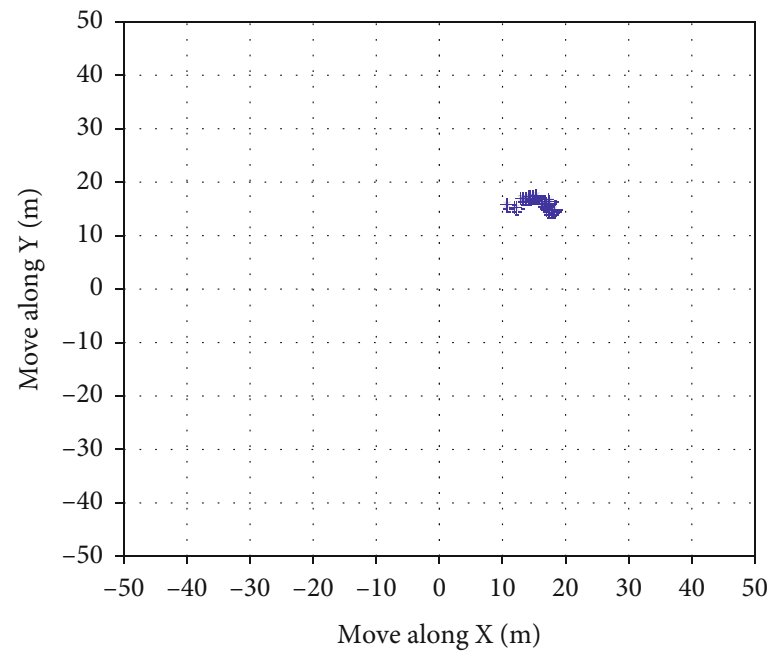

(d)

FIGURE 3: Relative coordinates of updated and optimized footprints to those given in the first version GEDI data: (a) optimized footprints of the first date; (b) optimized footprints of the second date; (c) updated footprints of the first date; (d) updated footprints of the second date. Color in $(a, b)$ indicates the number of GEDI footprints within the grid.

$12.9 \mathrm{~m}$ for the first date data and $30.5 \mathrm{~m}$ to $9.4 \mathrm{~m}$ for the second date data.

The effects of geolocations on the estimation of forest AGB are shown in Table 1 and Figure 4. In case III, the nominal geolocations are shifted $-12 \mathrm{~m}$ and $20 \mathrm{~m}$ along the $x$-axis and $y$-axis, respectively, for the first date data, and $24 \mathrm{~m}$ and $18 \mathrm{~m}$ along the $x$-axis and $y$-axis, respectively, for the second date data. Table 1 shows estimation models of forest $\mathrm{AGB}$ using GEDI waveform metrics developed by referencing to G-LiHT points extracted by different geolocations. Figure 4 is the corresponding scattering plots. The nominal geolocation, i.e., case I, gives the worst accuracy among all cases with coefficients of determination (i.e., $R^{2}$ ) of $0.48,0.56$, and 0.47 for models developed on RH, RHT, and WP, respectively. The model accuracy is substantially improved by the updated geolocations. $R^{2}$ in case II have been improved to $0.68,0.77$, and 0.64 for models developed on RH, RHT, and WP, respectively. $R^{2}$ in case III have been improved to $0.69,0.78$, and 0.65 for models developed on $\mathrm{RH}, \mathrm{RHT}$, and WP, respectively. Correspondingly, root mean square errors (RMSE) are decreased from $19.7 \mathrm{Mg} / \mathrm{ha}$, 18.1 Mg/ha, and $19.7 \mathrm{Mg} / \mathrm{ha}$ to $14.6 \mathrm{Mg} / \mathrm{ha}, 12.4 \mathrm{Mg} / \mathrm{ha}$, and $15.5 \mathrm{Mg} /$ ha for RH, RHT, and WP, respectively. Statistically, the updated and optimized geolocations have about the same estimation accuracy for the model developed on the same metrics.

4.2. Effects of Signal Start and Ending Points of Waveforms. Table 1 and Figure 4 show the results based on the first group of GEDI L2A products, i.e., a1. Table 2 shows results based on the other five groups of GEDI L2A products using case II and case III geolocations, i.e., a2 to a6. It is apparent that slope-adaptive metrics, i.e., RHT, perform the best on the estimations of forest AGB among three types of metrics in all six groups from a1 to a6. Taking a2 of case III as an example, $R^{2}$ of the model based on $\mathrm{RH}$ is 0.68 and that of 


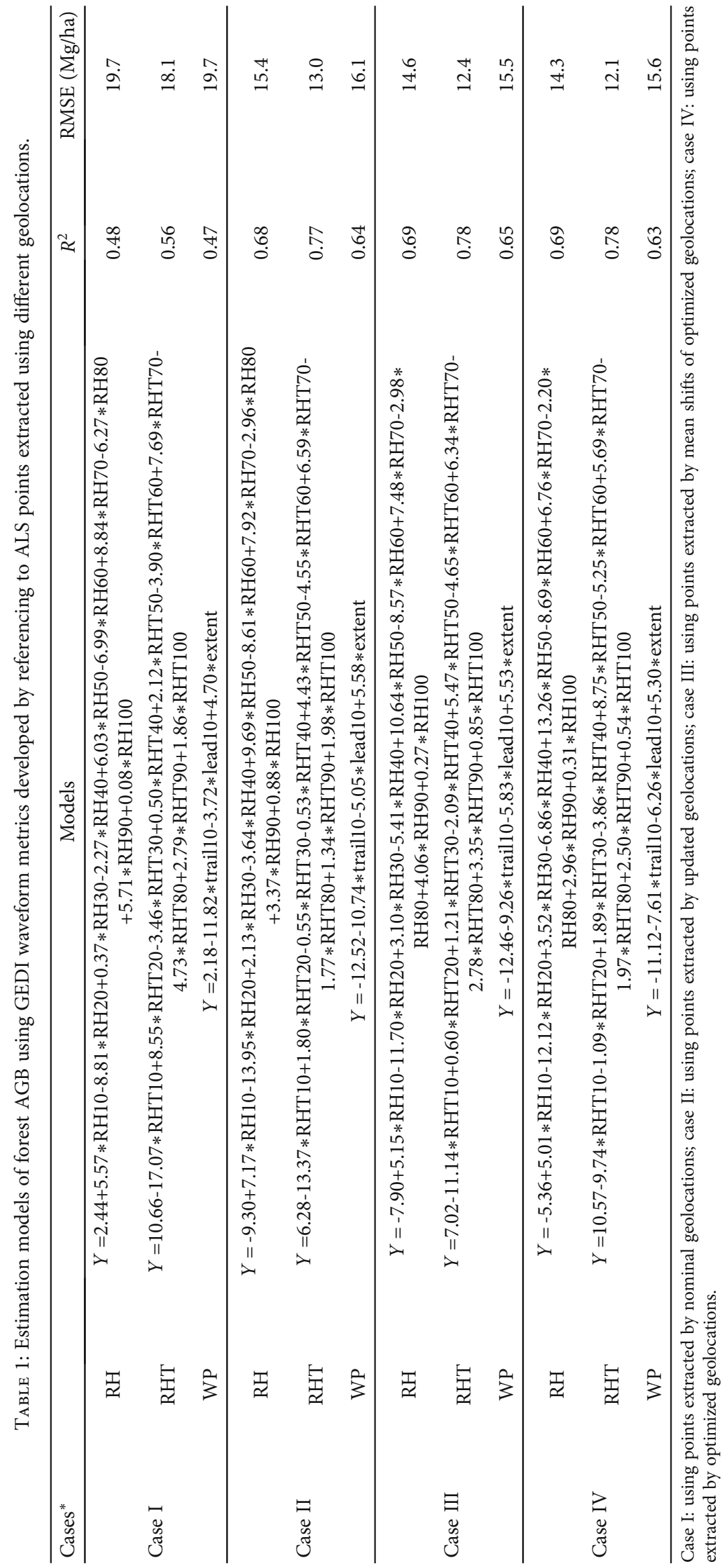




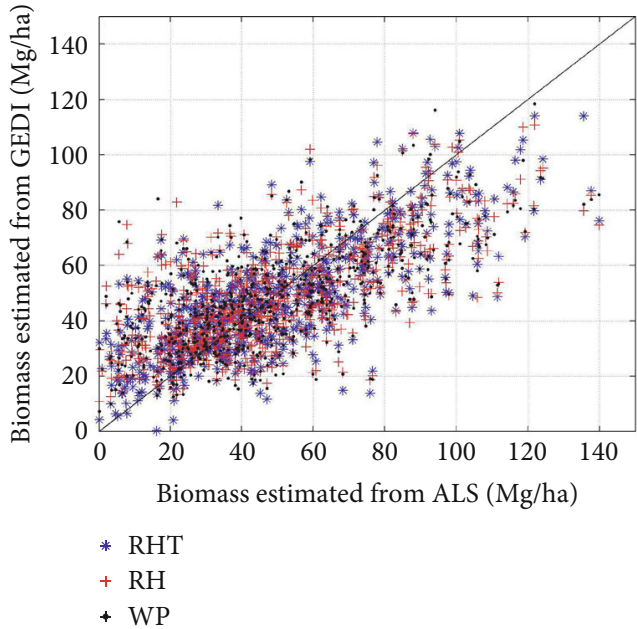

(a)

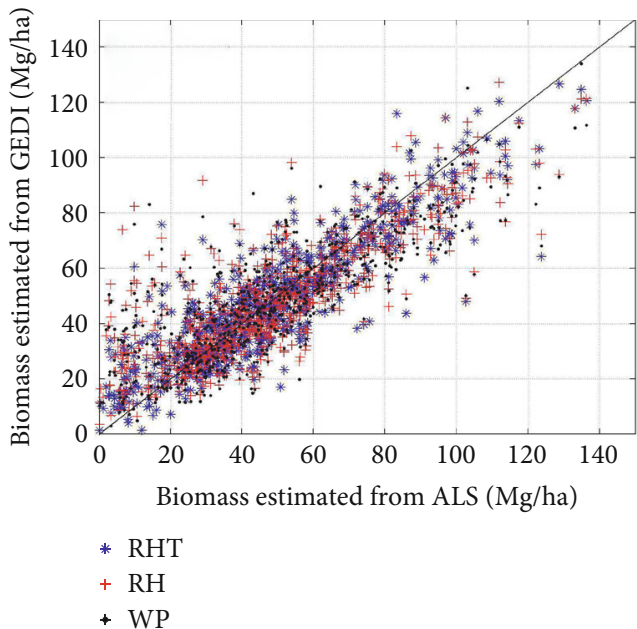

(c)

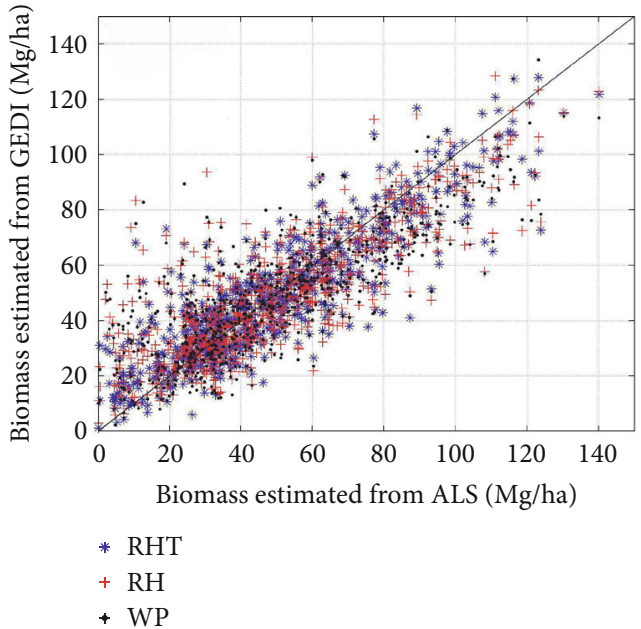

(b)

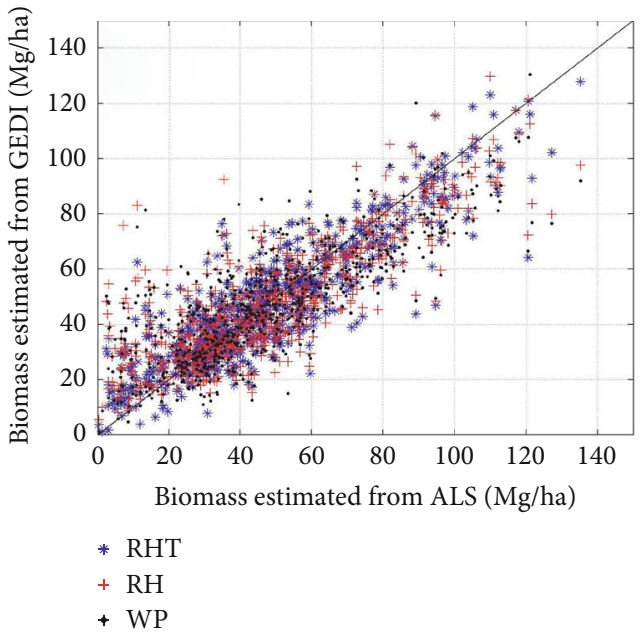

(d)

FIGURE 4: Scatter plots of forest AGB from ALS against those estimated from GEDI waveforms with different geolocations: (a) nominal geolocations, case I; (b) updated geolocations, case II; (c) nominal geolocations adjusted by mean shifts, case III; (d) nominal geolocations adjusted by detailed shifts, case IV.

RHT is 0.79 . The RMSE are $14.9 \mathrm{Mg} / \mathrm{ha}$ and $12.1 \mathrm{Mg} / \mathrm{ha}$ for $\mathrm{RH}$ and RHT, respectively.

The differences of six groups of the signal start and ending points on the estimation of forest AGB can easily be noticed in both case II and case III, especially for groups a4 and a5, although weaker than those between RHT and the other two metrics. In terms of metrics of RH and RHT, group a 4 gives the worst estimation accuracy, while the differences among the other five groups are quite weak. For models based on RH metrics in case III, $R^{2}$ of group a4 is 0.64 and the other five groups are around 0.67-0.69. For models based on RHT metrics in case III, $R^{2}$ of group a4 is about 0.74 and those of the other five groups are around 0.78-0.79. The situation of WP metrics is different from those of RH and RHT. Group a5 statistically gives the worst estimation accuracy. Its $R^{2}$ is 0.61 , and those of the other five groups are around $0.63-0.65$ in case III. This phenomenon can also be observed in case II, although $R^{2}$ is about
0.1 02 lower than the corresponding ones in case III. This can be interpreted by different noise thresholds. Group a4 used the maximum threshold in the identification of both signal start and ending points, i.e., six times the standard deviation of noise. That means group a 4 gives the highest elevation of signal ending points and the shortest waveform length. Group a5 used the minimum threshold in the identification of the signal ending point, i.e., two times the standard deviation of noise. That means group a5 gives the lowest elevation of signal ending point and the longest waveform length. Therefore, the effects of signal start and ending points cannot be neglected. It should be very careful if metrics of group a4 and group a5 are used.

4.3. Effects of Terrain Conditions. The external terrain slope is necessary for the calculation of slope-adaptive metrics as shown in Equations (1)-(6). Figure 5 shows scatter plots of terrain slopes extracted from G-LiHT ALS data against 


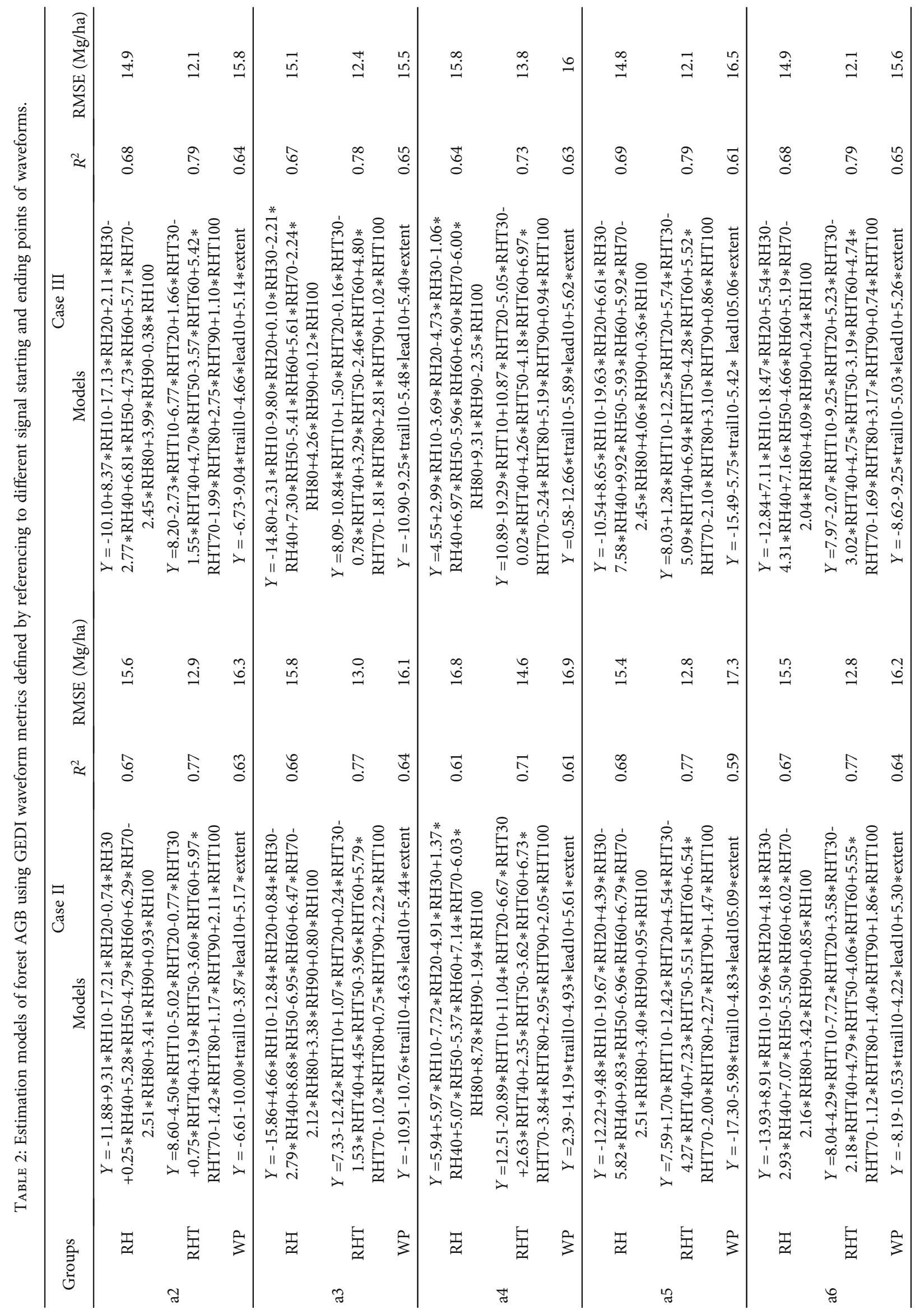




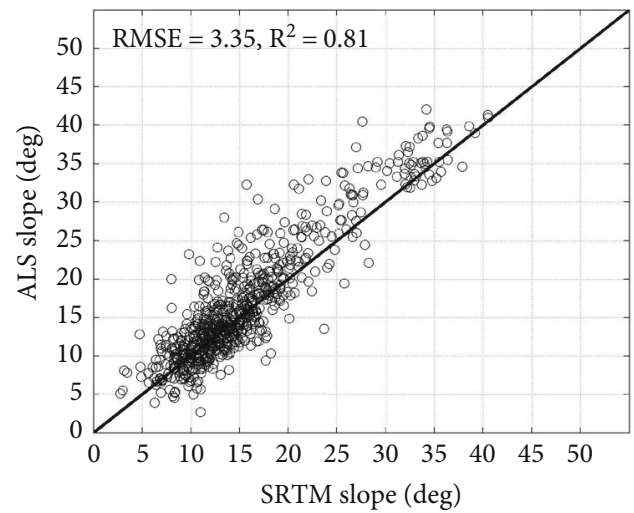

- Nominal geolocation

(a)

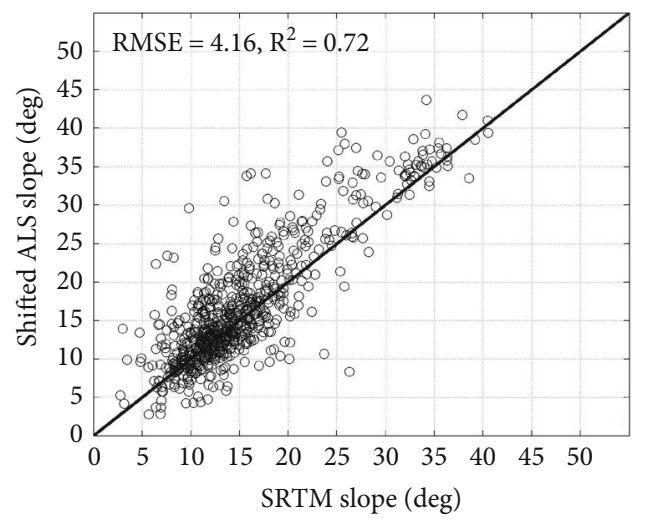

- Nominal geolocations

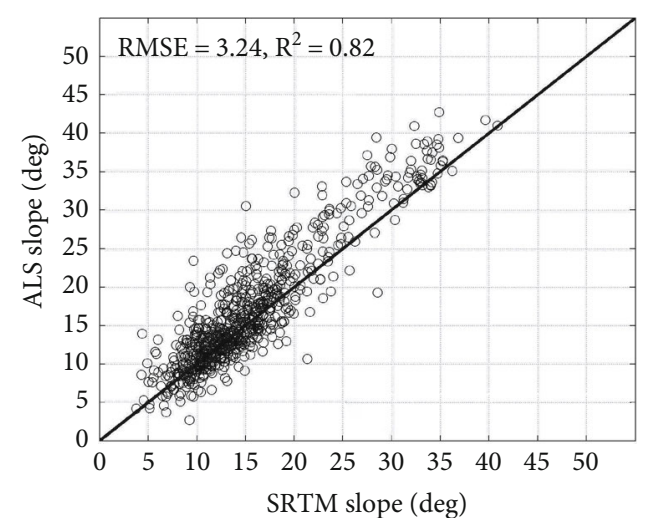

- Updated geolocation

(b)

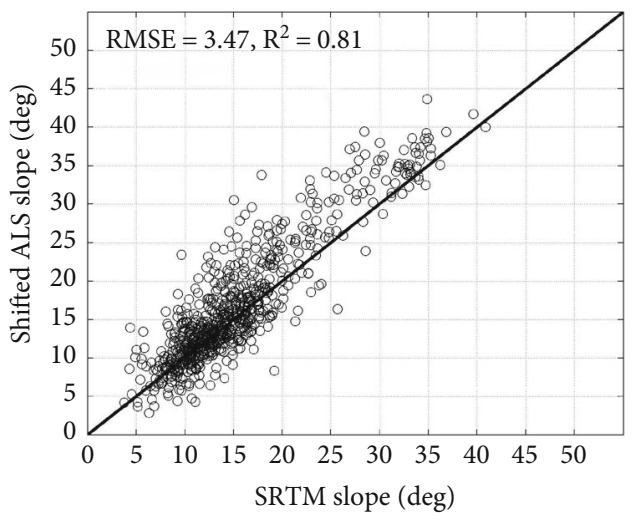

- Updated geolocation

(d)

FIGURE 5: Comparisons of terrain slopes extracted from SRTM DEM and ALS DEM using nominal, updated, and optimized geolocations: (a) ALS against SRTM using nominal geolocations, i.e., case I; (b) ALS against SRTM using updated geolocations, i.e., case II; (c) optimized ALS against nominal SRTM; (d) optimized ALS against updated SRTM.

SRTM DEM over GEDI footprints using different geolocations. Figure 5(a) uses terrain slopes extracted by the nominal geolocations from SRTM and ALS, respectively, while Figure 5(b) is the same as Figure 5(a) but extracted by updated geolocations. These two figures show the uncertainty of terrain slopes extracted from SRTM by taking those from ALS as references. It can be seen that they have about the same accuracy with RMSE of about $3^{\circ}$. Figure 5(c) shows terrain slopes extracted from SRTM using the nominal geolocations but against those from ALS using optimized geolocations. It can be seen from Figures 5(a) and 5(c) that the determination coefficients $\left(R^{2}\right)$ decreased from 0.81 to 0.72 and the RMSE increased from $3.35^{\circ}$ to $4.16^{\circ}$, which indicates that errors caused by geolocation uncertainties cannot be ignored if the first version GEDI data product is used. Figure 5(d) shows the terrain slopes extracted from SRTM using the updated geolocations against those from ALS using optimized geolocations. It can be seen that the difference between Figures 5(b) and 5(d) is quite weak, with determination coefficients $\left(R^{2}\right)$ decreased from 0.82 to 0.81 and RMSE increased from $3.24^{\circ}$ to $3.47^{\circ}$. This indicates that the geolocation uncertainty in the second version GEDI products can statistically be ignored, at least in the test site of this study.

Table 3 further shows estimation models of forest AGB using GEDI waveform metrics under different terrain conditions. For terrain slopes extracted from SRTM by nominal geolocations, there are 447 and 297 GEDI footprints having terrain slopes below and above $15^{\circ}$, respectively. For footprints located on terrain slopes $\leq 15^{\circ}, R^{2}$ of estimation model based on RHT metrics is 0.6 with RMSE of $17.3 \mathrm{Mg} / \mathrm{ha}$. The scatter plot is shown in Figure 6(a). The difference between models based on different metrics is not quite obvious, although the RHT metrics give the best results in numbers. For footprints located on terrain slopes $>15^{\circ}, R^{2}$ of the estimation model based on RH, RHT, and WP metrics is 0.34 , 0.44 , and 0.31 , respectively, and the corresponding RMSE is $20.3 \mathrm{Mg} / \mathrm{ha}, 18.7 \mathrm{Mg} / \mathrm{ha}$, and $20.6 \mathrm{Mg} / \mathrm{ha}$, respectively. The scatter plot is shown in Figure 6(b). Their estimation accuracy is quite low, although the model based on RHT metrics gives the best results. The performance of these metrics on the estimation of forest $A G B$ is substantially improved if the ALS data are extracted using the updated geolocation, i.e., case II. For footprints located on terrain 


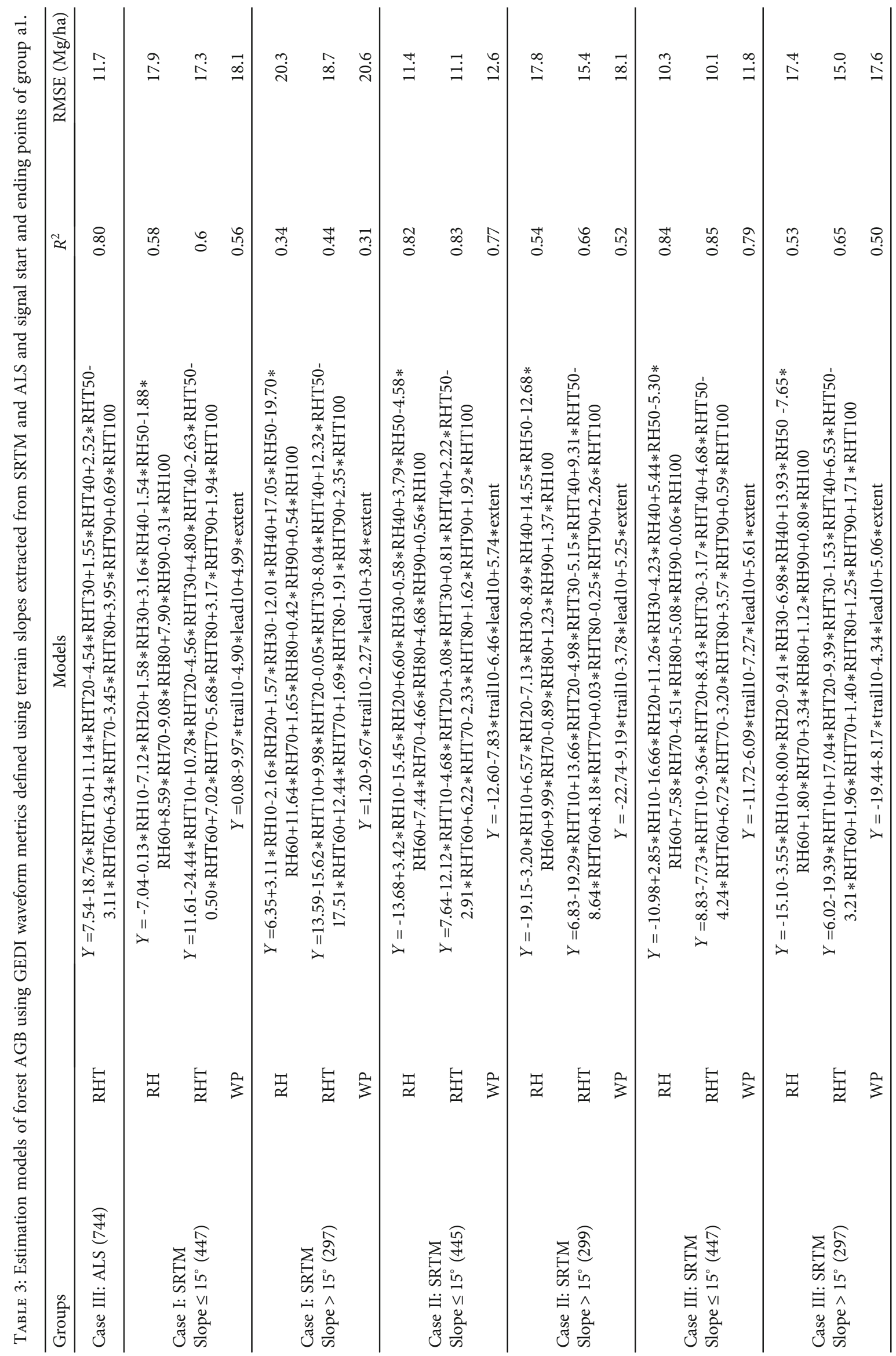




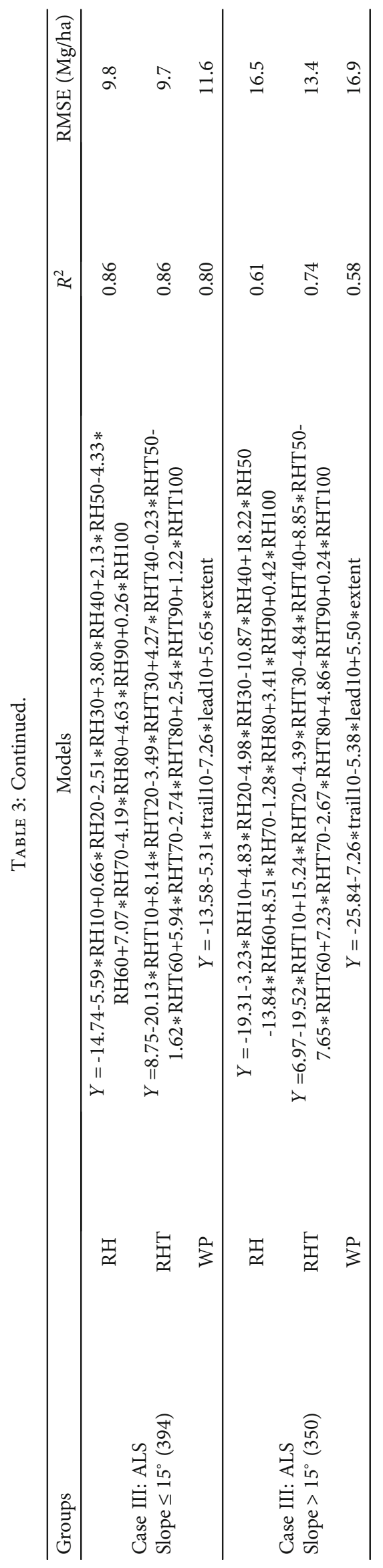



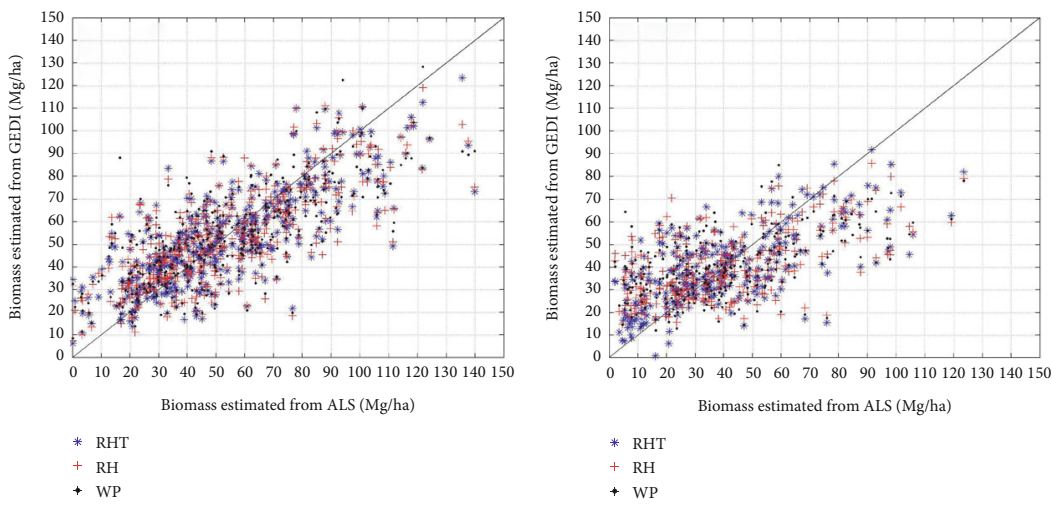

(a)

(b)
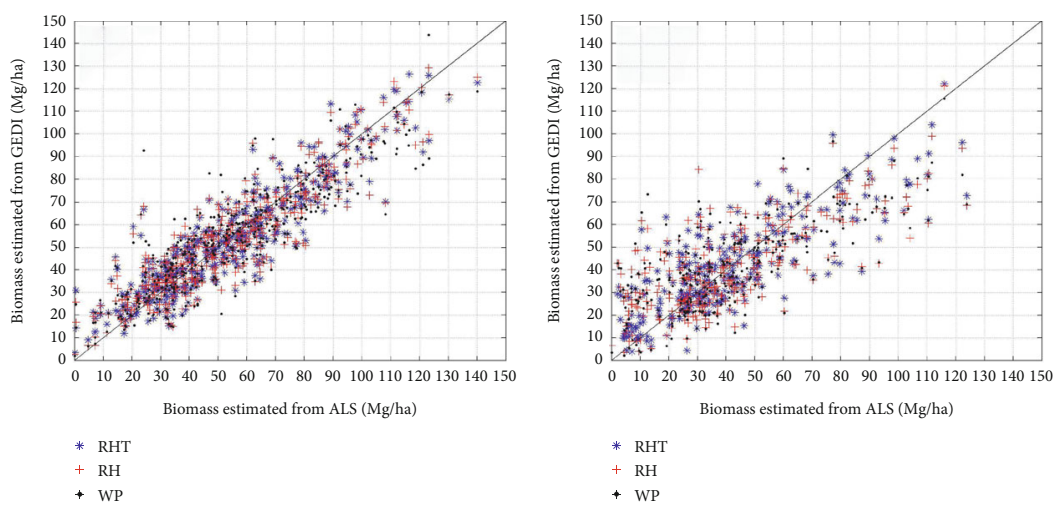

(c)

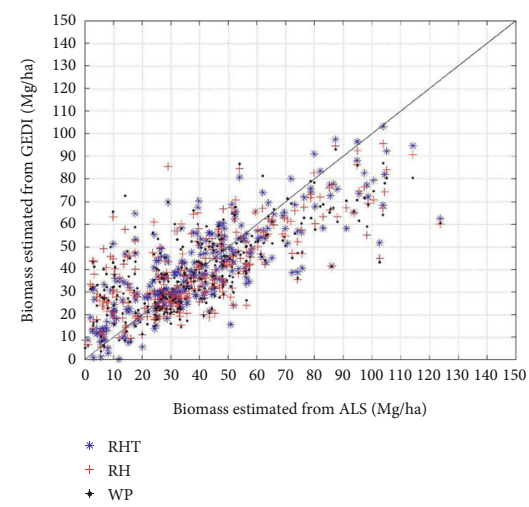

(f)
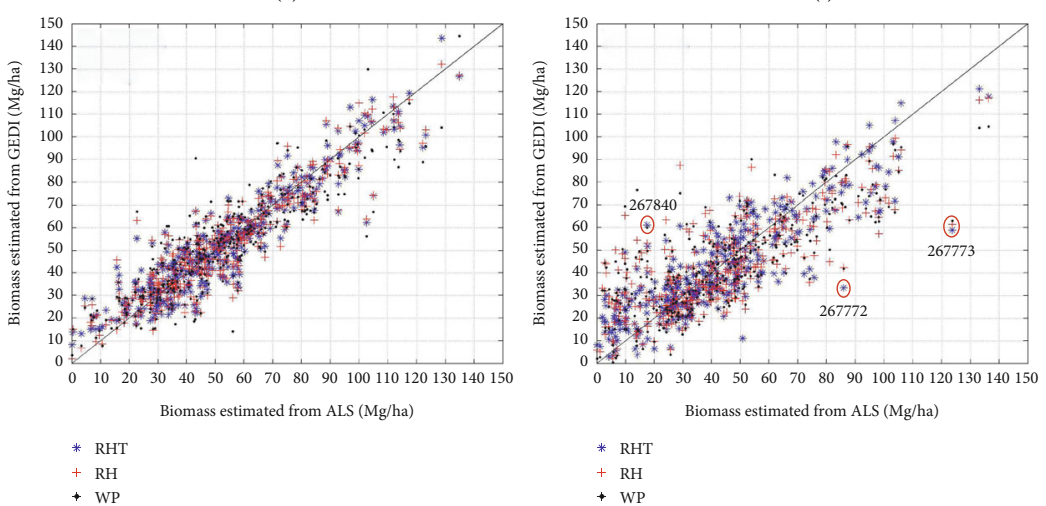

(g)

(h)

FIGURE 6: Scatter plots of forest AGB from ALS against those from GEDI waveforms with different terrain slopes: (a, b) use slopes $\leq 15^{\circ}$ and $>15^{\circ}$ extracted from SRTM by nominal geolocation, i.e., case I; (c, d) use slopes $\leq 15^{\circ}$ and $>15^{\circ}$ extracted from SRTM by updated geolocation, i.e., case II; (e, f) use slopes $\leq 15^{\circ}$ and $>15^{\circ}$ extracted from SRTM by optimized geolocation, i.e., case III; (g, h) use slopes $\leq 15^{\circ}$ and $>15^{\circ}$ extracted from ALS $\leq 15^{\circ}$ with optimized geolocation. 


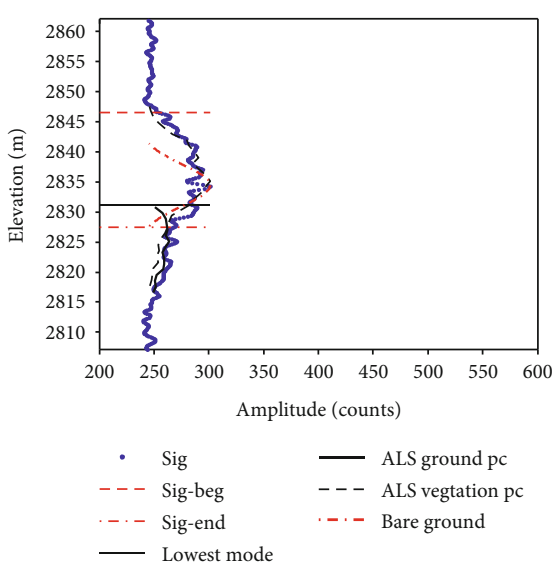

(a)

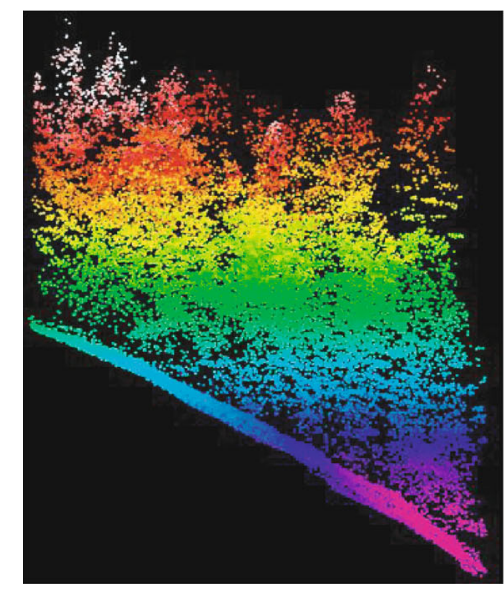

(d)

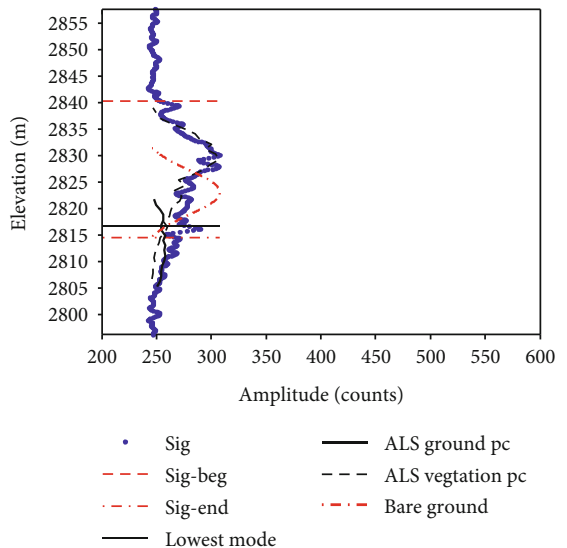

(b)

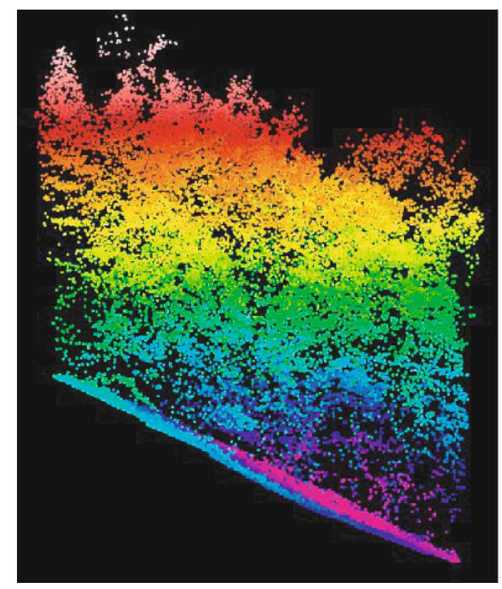

(e)

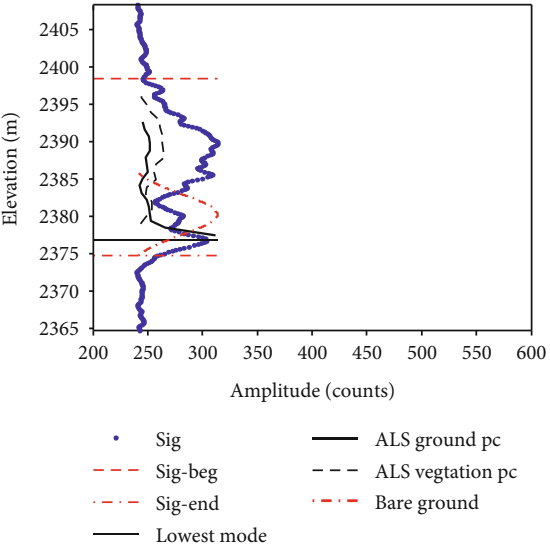

(c)

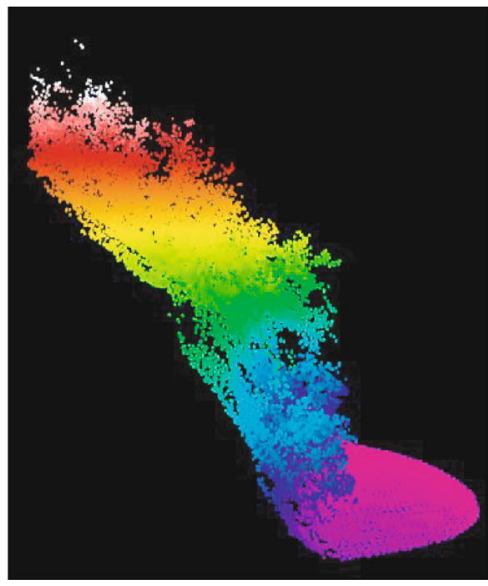

(f)

FIGURE 7: Typical waveforms and corresponding point cloud: (a-c) are waveforms of footprint 267772, 267773, and 267840; (d-f) are point clouds corresponding to $(\mathrm{a}-\mathrm{c})$.

slopes $\leq 15^{\circ}, R^{2}$ of the estimation model based on RHT metrics is increased from 0.6 to 0.83 and RMSE is decreased from $17.3 \mathrm{Mg} / \mathrm{ha}$ to $11.1 \mathrm{Mg} / \mathrm{ha}$. The scatter plot is shown in Figure 6(c). The model based on RH metrics gives about the same estimation accuracy as that on RHT, and the model based on WP metrics gives the worst estimation accuracy with $R^{2}=0.77$ and $\mathrm{RMSE}=12.6 \mathrm{Mg} / \mathrm{ha}$. For footprints located on terrain slopes $>15^{\circ}$, the estimation model based on RHT metrics gives the best accuracy with $R^{2}$ increased from 0.44 to 0.66 and RMSE decreased from $18.7 \mathrm{Mg} / \mathrm{ha}$ to $15.4 \mathrm{Mg} / \mathrm{ha}$. The scatter plot is shown in Figure 6(d). The $\mathrm{RH}$ and WP metrics give approximately the same estimation accuracy with $R^{2}$ of 0.54 and 0.52 and RMSE of $17.8 \mathrm{Mg} / \mathrm{ha}$ and $18.1 \mathrm{Mg} / \mathrm{ha}$, respectively.

It can also be seen in Table 3 and Figures 6(e) and 6(f) that the difference between updated and optimized geolocations on the estimation of forest AGB is quite weak. Their determination coefficients are 0.83 and 0.85 for RHT metrics using footprints with terrain slopes $\leq 15^{\circ}$, respectively. For terrain slopes $>15^{\circ}$, they are 0.66 and 0.65 , respectively. The improvement of estimation accuracy of forest AGB using exact terrain slopes from ALS is weak for footprints located on terrain slopes $\leq 15^{\circ}$ as shown in Table 3 and
Figure 6(g). However, the situation is different for footprints located on terrain slopes $>15^{\circ} . R^{2}$ of models based on RHT metrics is further improved from 0.65 to 0.74 , and RMSE is further decreased from $15.0 \mathrm{Mg} /$ ha to $13.4 \mathrm{Mg} / \mathrm{ha}$. The scatter plot is shown in Figure 6(h).

Three typical waveforms are labeled in Figure 6(h), i.e., footprints of 267840, 267772, and 267773. The forest AGB of waveforms 267772 and 267773 are obviously underestimated while that of 267840 is obviously overestimated. Figure 7 shows their waveforms and corresponding point cloud. It can be seen that the underestimation of 267772 and 267773 is caused by the error on the identification of signal ending points. For footprint 267772, the ALS shows that the elevation of signal ending point should be about $2818 \mathrm{~m}$, while that of the identified ending point is about $2827 \mathrm{~m}$. For footprint of 267773 , the lowest elevation of ALS and that of identified ending point is about $2808 \mathrm{~m}$ and 2815 , respectively. The misidentification of these two points should be deduced to the weak penetration capabilities.

According to the definition of slope-adaptive metrics, the ground surface is modeled as an inclined plane with a given terrain slope. Naturally, performance of slope- 
adaptivemetrics should be bad if the ground surface cannot be modeled as an inclined plane. It can be seen that the footprint of 267840 is exactly such a case as shown in Figures 7(c) and 7(f). The terrain within the footprint is too complicated. The lower part is flat while the middle part has a larger slope than the upper part. These three parts cannot be modeled by a simple plane. The reference forest AGB of this footprint and the estimation by RHT, $\mathrm{RH}$, and WP metrics are $17.42 \mathrm{Mg} / \mathrm{ha}, 61.2 \mathrm{Mg} / \mathrm{ha}, 60 \mathrm{Mg} / \mathrm{ha}$, and $49.8 \mathrm{Mg} / \mathrm{ha}$, respectively. Therefore, the forest AGB is severely overestimated by all three types of metrics. None of the three types of metrics are effective for the estimation of forest AGB from such kinds of waveforms.

\section{Discussions}

The shift determined by matches between simulated and GEDI waveforms is just a kind of guess. We have no way to exactly evaluate the real geolocation accuracy of GEDI waveforms in this study. The shift distance may be not very exact, but the obvious improvement of estimation accuracy of forest AGB is clear, and the updated geolocation in the second version of GEDI products confirmed the geolocation error of the first version GEDI footprints. The mean shift distance is approximately at the same level as the footprint size. Therefore, the geolocation error needs to be considered in linking GEDI waveforms with other sources of reference data, especially when field measurements are involved and the first version GEDI is used.

There is no problem in refining the geolocation of GEDI data using the ALS point cloud as we did in this study. It is challenging to identify geolocation errors of GEDI data when ALS data is unavailable. The results of this study show that the mean shift distance is at approximately the same level as the pixel size of SRTM DEM, ASTER GDEM, and AW3D30. Therefore, it is difficult to refine geolocation just by shifting footprints along or across tracks on these datasets as in previous studies [33]. In the last decades, the studies on stereo imagery acquired by cameras onboard unmanned aerial vehicles (UAV) grew rapidly [34]. It has been demonstrated that UAV stereo imagery has good performance on the depiction of forest canopy top if the stereo images are acquired by proper settings [35]. In addition, the highresolution spaceborne stereo imagery acquired by Worldview satellites with proper observation geometry also can make a good description of the forest canopy top [36]. Theoretically, the position of the signal ending point is easily affected by completed terrain conditions and multiple scattering within a forest, while the signal start point should be more stable. Therefore, optical stereo imagery acquired by UAV or high-resolution spaceborne sensors may be used for the optimization of GEDI data, especially over mountainous areas.

The outstanding performance of slope-adaptive metrics has been demonstrated over mountainous areas. However, its weakness has also been revealed. Slope-adaptive metrics are improper if the terrain condition is too complicated or the ground surface cannot be modeled as an inclined plane. Therefore, slope-adaptive metrics tend to fail for footprints located on valleys, ridges, or partially covered on Ghaut. In addition, slope-adaptive metrics are easily affected by the signal ending points. For dense forests standing on a steep hillside, a smaller threshold should be used in the identification of signal ending points. For sparse or medium forests on flat terrains, a bigger threshold should be used in the identification of signal ending points in order to exclude the influences of multiple scattering. It may be useful to classify GEDI waveforms and select proper signal ending points accordingly.

\section{Conclusions}

The performance of GEDI data on estimations of forest AGB over mountainous areas has been assessed using 744 waveforms with terrain slopes up to $45^{\circ}$. Three types of waveform metrics are employed in the model development, including slope-adaptive metrics (i.e., RHT), relative height metrics defined by referencing to the lowest mode of waveform (i.e., RH), and waveform parameters (i.e., WP). It can be concluded from this study that

(1) there is an obvious shift in the first version geolocations of GEDI waveforms, at least for datasets used in this study. The footprints shifted to the left forward of nominal geolocations with a distance of about $24 \mathrm{~m} \sim 30 \mathrm{~m}$. The geolocation shifts are substantially corrected in the second version. The coefficients of determination $\left(R^{2}\right)$ of models based on RHT metrics are improved from 0.56 to 0.77 with root mean square error (RMSE) decreased from $18.1 \mathrm{Mg} / \mathrm{ha}$ to $13.0 \mathrm{Mg} / \mathrm{ha}$

(2) some differences are observed in the estimation of forest AGB using metrics defined by different signal starting and ending points. Group a 4 gives the worst estimation accuracy for metrics of RH and RHT, because it gives the shortest effective waveforms due to the maximum threshold used in the identification of signal starting and ending points. Group a5 gives the worst estimation accuracy for metrics of WP, because it gives the longest effective waveforms due to the minimum threshold used in the identification of signal starting and ending points

(3) all three types of waveform metrics have a good performance on the estimation of forest AGB using footprints on terrain slope $\leq 15^{\circ}$ so long as the geolocation shift is fixed. $R^{2}$ of models based on $\mathrm{RH}$, RHT, and WP metrics are $0.82,0.83$, and 0.77 with RMSE of $11.4 \mathrm{Mg} / \mathrm{ha}, 11.1 \mathrm{Mg} / \mathrm{ha}$, and $12.6 \mathrm{Mg} / \mathrm{ha}$, respectively. The outstanding performance of slopeadaptive metrics is clearly exhibited on the estimation of forest AGB using footprints on terrain slope $>15^{\circ}$. $R^{2}$ of models based on RH, RHT, and WP metrics are $0.54,0.66$, and 0.52 with RMSE of $17.8 \mathrm{Mg} / \mathrm{ha}$, $15.4 \mathrm{Mg} / \mathrm{ha}$, and $18.1 \mathrm{Mg} / \mathrm{ha}$, respectively. $R^{2}$ of models based on RHT metrics can be further improved to 0.74 with RMSE of $13.4 \mathrm{Mg} / \mathrm{ha}$ if the 
terrain slopes are extracted from ALS data. For all 744 waveforms, the model based on RHT metrics gives the best estimation accuracy with $R^{2}$ of 0.8 and RMSE of 11.7 if ALS terrain slopes are used

As a summary, the GEDI waveforms with second version geolocations have good performance on the estimation of forest AGB, especially for footprints on terrain slopes $\leq 15^{\circ}$. Compared with $\mathrm{RH}$ and WP metrics, the slopeadaptive metrics have better estimation accuracy of forest AGB over mountainous areas, especially when ALS terrain slopes are used. It has to be pointed out that the performance of slope-adaptive metrics on the estimation of forest AGB is limited if the terrain conditions within the footprint are too complicated. The global products of slope-adaptive metrics will be publically released as supplementary components to the product list defined by the GEDI science team.

\section{Data Availability}

The data used to support the findings of this study are publicly available. GEDI data can be downloaded from the website of NASA's Land Processes Distributed Active Archive Center (LP DAAC) located at the USGS Earth Resources Observation and Science (EROS) Center (https://e4ftl01.cr .usgs.gov/GEDI), while G-LiHT data can be found online at https://glihtdata.gsfc.nasa.gov/files/G-LiHT/.

\section{Conflicts of Interest}

The authors declare that there are no conflicts of interest regarding the publication of this article.

\section{Authors' Contributions}

WN processed and analyzed the data and drafted the manuscript, ZZ helped with the experiment design and manuscript preparation, and GS revised the manuscript.

\section{Acknowledgments}

This study was sponsored by the National Key R\&D Program of China (2020YFE0200800 and 2017YFA0603002) and National Natural Science Foundation of China (Grant No. 42022009). Special thanks are due to the G-LiHT team for the open-access ALS point cloud and GEDI team for the public release of data products.

\section{References}

[1] P. Friedlingstein, M. W. Jones, M. O'Sullivan et al., "Global carbon budget 2019," Earth System Science Data, vol. 11, no. 4, pp. 1783-1838, 2019.

[2] S. S. Saatchi, N. L. Harris, S. Brown et al., "Benchmark map of forest carbon stocks in tropical regions across three continents," Proceedings of the National Academy of Sciences of the United States of America, vol. 108, no. 24, pp. 9899-9904, 2011.

[3] A. Baccini, W. Walker, L. Carvalho, M. Farina, D. SullaMenashe, and R. A. Houghton, "Tropical forests are a net car- bon source based on aboveground measurements of gain and loss," Science, vol. 358, no. 6360, pp. 230-234, 2017.

[4] N. L. Harris, S. Brown, S. C. Hagen et al., "Baseline map of carbon emissions from deforestation in tropical regions," Science, vol. 336, no. 6088, pp. 1573-1576, 2012.

[5] X. P. Song, C. Huang, S. S. Saatchi, M. C. Hansen, and J. R. Townshend, "Annual carbon emissions from deforestation in the Amazon basin between 2000 and 2010," PLoS One, vol. 10, no. 5, article e0126754, 2015.

[6] V. Avitabile, M. Herold, G. B. M. Heuvelink et al., "An integrated pan-tropical biomass map using multiple reference datasets," Global Change Biology, vol. 22, no. 4, pp. 14061420, 2016.

[7] F. G. Hall, K. Bergen, J. B. Blair et al., "Characterizing 3D vegetation structure from space: mission requirements," Remote Sensing of Environment, vol. 115, no. 11, pp. 2753-2775, 2011.

[8] M. C. Hansen, P. Potapov, and A. Tyukavina, "Comment on "tropical forests are a net carbon source based on aboveground measurements of gain and loss"," Science, vol. 363, no. 6423, article eaar3629, 2019.

[9] S. Quegan, T. le Toan, J. Chave et al., "The European Space Agency BIOMASS mission: measuring forest above-ground biomass from space," Remote Sensing of Environment, vol. 227, pp. 44-60, 2019.

[10] T. le Toan, S. Quegan, M. W. J. Davidson et al., "The BIOMASS mission: mapping global forest biomass to better understand the terrestrial carbon cycle," Remote Sensing of Environment, vol. 115, no. 11, pp. 2850-2860, 2011.

[11] R. Dubayah, J. B. Blair, S. Goetz et al., "The Global Ecosystem Dynamics Investigation: High-resolution laser ranging of the Earth's forests and topography," Science of Remote Sensing, vol. 1, p. 100002, 2020.

[12] W. L. Qi, S. K. Lee, S. Hancock et al., "Improved forest height estimation by fusion of simulated GEDI Lidar data and TanDEM-X InSAR data," Remote Sensing of Environment, vol. 221, pp. 621-634, 2019.

[13] R. Mitsuhashi, J. Murooka, D. Sakaizawa et al., "Overview of vegetation Lidar "MOLI"," in Sensors, Systems, and NextGeneration Satellites XXII, vol. 10785, October 2018.

[14] S. Du, L. Liu, X. Liu, X. Zhang, X. Gao, and W. Wang, "The solar-induced chlorophyll fluorescence imaging spectrometer (SIFIS) onboard the first Terrestrial Ecosystem Carbon Inventory Satellite (TECIS-1): specifications and prospects," Sensors, vol. 20, no. 3, p. 815, 2020.

[15] X. M. Tang, J. F. Xie, R. Liu et al., "Overview of the GF-7 laser altimeter system mission," Earth and Space Science, vol. 7, no. $1,2020$.

[16] M. Price, G. Gratzer, L. A. Duguma, T. Kohler, D. Maselli, and R. Romeo, "Mountain Forests in a Changing World - Realising Values, Addressing Challenges.," 2011, https://www.perth.uhi .ac.uk/subject-areas/centre-for-mountain-studies/researchthemes/sustainable-mountain-development-andcharacterising-mountains/mountain-forests-in-a-changingworld/.

[17] M. Lundbäck, H. Persson, C. Häggström, and T. Nordfjell, "Global analysis of the slope of forest land," Forestry: An International Journal of Forest Research, vol. 94, no. 1, pp. 54-69, 2020.

[18] M. A. Lefsky, "A global forest canopy height map from the Moderate Resolution Imaging Spectroradiometer and the 
Geoscience Laser Altimeter System," Geophysical Research Letters, vol. 37, no. 15, p. L15401, 2010.

[19] S. Lee, W. Ni-Meister, W. Yang, and Q. Chen, "Physically based vertical vegetation structure retrieval from ICESat data: validation using LVIS in White Mountain National Forest, New Hampshire, USA," Remote Sensing of Environment, vol. 115, no. 11, pp. 2776-2785, 2011.

[20] J. J. van Zyl, “The Shuttle Radar Topography Mission (SRTM): a breakthrough in remote sensing of topography," Acta Astronautica, vol. 48, no. 5-12, pp. 559-565, 2001.

[21] N. Chrysoulakis, M. Abrams, Y. Kamarianakis, and M. Stanislawski, "Validation of ASTER GDEM for the area of Greece," Photogrammetric Engineering and Remote Sensing, vol. 77, no. 2, pp. 157-165, 2011.

[22] D. Yamazaki, D. Ikeshima, R. Tawatari et al., "A high-accuracy map of global terrain elevations," Geophysical Research Letters, vol. 44, no. 11, pp. 5844-5853, 2017.

[23] B. Wessel, M. Huber, C. Wohlfart, U. Marschalk, D. Kosmann, and A. Roth, "Accuracy assessment of the global TanDEM-X Digital Elevation Model with GPS data," ISPRS Journal of Photogrammetry and Remote Sensing, vol. 139, pp. 171-182, 2018.

[24] Y. Wang, W. Ni, G. Sun, H. Chi, Z. Zhang, and Z. Guo, "Slope-adaptive waveform metrics of large footprint lidar for estimation of forest aboveground biomass," Remote Sensing of Environment, vol. 224, pp. 386-400, 2019.

[25] B. D. Cook, L. Corp, R. Nelson et al., "NASA Goddard's LiDAR, Hyperspectral and Thermal (G-LiHT) airborne imager," Remote Sensing, vol. 5, no. 8, pp. 4045-4066, 2013.

[26] R. Nelson, H. Margolis, P. Montesano et al., "Lidar-based estimates of aboveground biomass in the continental US and Mexico using ground, airborne, and satellite observations," Remote Sensing of Environment, vol. 188, pp. 127-140, 2017.

[27] P. Potapov, X. Li, A. Hernandez-Serna et al., "Mapping global forest canopy height through integration of GEDI and Landsat data," Remote Sensing of Environment, vol. 253, p. 112165, 2021.

[28] D. J. Harding and C. C. Carabajal, "ICESat waveform measurements of within-footprint topographic relief and vegetation vertical structure," Geophysical Research Letters, vol. 32, p. L21S10, 2005.

[29] S. Hancock, P. Lewis, M. Foster, M. Disney, and J. P. Muller, "Measuring forests with dual wavelength lidar: a simulation study over topography," Agricultural and Forest Meteorology, vol. 161, pp. 123-133, 2012.

[30] T. Tachikawa, M. Hato, M. Kaku, and A. Iwasaki, "Characteristiacs of ASTERGDEM version 2," in Presented at the IEEE International Geoscience and Remote Sensing Symposium (IGARSS), Vancouver, Canada, 2011.

[31] M. Zink, M. Bachmann, B. Brautigam et al., "TanDEM-X: the new global DEM takes shape," IEEE Geoscience and Remote Sensing Magazine, vol. 2, no. 2, pp. 8-23, 2014.

[32] T. Tadono, H. Ishida, F. Oda, S. Naito, K. Minakawa, and H. Iwamoto, "Precise global DEM generation by ALOS PRISM," ISPRS Ann. Photogramm. Remote Sens. Spatial Inf. Sci., vol. II-4, pp. 71-76, 2014.

[33] G. Sun, K. J. Ranson, V. I. Kharuk, and K. Kovacs, "Validation of surface height from shuttle radar topography mission using shuttle laser altimeter," Remote Sensing of Environment, vol. 88, no. 4, pp. 401-411, 2003.

[34] W. J. Ni, J. C. Dong, G. Q. Sun et al., "Synthesis of leaf-on and leaf-off unmanned aerial vehicle (UAV) stereo imagery for the inventory of aboveground biomass of deciduous forests," Remote Sensing, vol. 11, no. 7, p. 889, 2019.

[35] W. J. Ni, G. Q. Sun, Y. Pang et al., "Mapping threedimensional structures of forest canopy using UAV stereo imagery: evaluating impacts of forward overlaps and image resolutions with LiDAR data as reference," IEEE Journal of Selected Topics in Applied Earth Observations and Remote Sensing, vol. 11, no. 10, pp. 3578-3589, 2018.

[36] P. M. Montesano, C. Neigh, G. Sun, L. Duncanson, J. van den Hoek, and K. J. Ranson, "The use of sun elevation angle for stereogrammetric boreal forest height in open canopies," Remote Sensing of Environment, vol. 196, pp. 76-88, 2017. 